\title{
Optimal Primary-Secondary user Pairing and Power Allocation in Cognitive Cooperative Multiple Access Channels
}

\author{
Saygın Bakşi and Onur Kaya \\ Department of Electrical and Electronics Engineering \\ Işık University, Şile, Istanbul, Turkey \\ saygin.baksi@isik.edu.tr \\ onurkaya@isikun.edu.tr
}

\begin{abstract}
We develop jointly optimal power control and primary-secondary user partnering strategies for a cognitive cooperative multiple access channel with $K$ primary and $K$ secondary users. For each primary user, a cooperating secondary user is assigned. We consider both underlay and overlay modes for cognition/cooperation. In overlay mode, each secondary user decodes and relays part of its assigned primary user's message, and simultaneously transmits its own independent message, while ensuring the primary user achieves at least its single user capacity with power control. The encoding is based on channel adaptive block Markov superposition coding, where the powers assigned to primary and secondary user codewords are optimized so as to maximize either the system's sum rate, or the sum of secondary users' rates. In underlay mode, each secondary user employs independent signalling and allocates its power to maximize its own rate, without decreasing its assigned primary user's rate. The partnering problem for either mode is reduced to a maximum weighted matching (MWM) problem on a bipartite graph, and solved jointly optimally with the power allocation problem.
\end{abstract}

\section{INTRODUCTION}

Cognitive radio and user cooperation are two promising techniques for improving the user capacity and throughput performance of the next generation wireless networks. The foundations of user cooperation date back to as early as 1970s, when the relay channel was introduced [1]; whereas cognitive radio, whose initial intended application was the opportunistic use of licensed spectrum by secondary users, is a newer concept introduced in [2]. Despite having emerged at different times from different needs, both techniques rely on the common idea of exploiting the advanced capabilities of smart transmitters and receivers, which are aware of their surroundings. Hence, it is quite natural to jointly design cognition and cooperation strategies in wireless communication setups, including the cognitive cooperative multiple access channel considered in this paper.

Cognitive radios, depending on their level of sophistication, can operate in a number of modes: they can detect the frequency voids in the spectrum and use them for their transmissions (interweave mode); they can transmit at a power level to satisfy a given interference temperature constraint at the primary users (underlay mode); or actively participate in the communication of the primary user (overlay mode) [3]. In this paper, we will mostly focus on the overlay mode, but will also obtain the optimal partner selection in a power controlled underlay setup as a benchmark.

In overlay cognitive radio, the secondary users may decode primary users' signals and use them while creating their codewords, or even relay the PU (primary user) messages to communicate their own messages under better conditions. Most of the existing information theoretic works on cognitive radio

This work was supported by TUBITAK grant 111E108. consider separate primary and secondary transmitter/receiver pairs, which results in an interference channel model [4]-[10]. However, these works apply a combination of complicated techniques such as rate splitting, Gelfand Pinsker (GP) binning etc. As a result, the obtained rate regions are too difficult to even characterize and compute, and do not lend themselves to generalization to larger systems with many users, or optimization in terms of the powers. Therefore, in this paper, we shall consider a simpler overlay cooperation model involving a multiple access setup with a common receiver instead.

While the most commonly known and widely studied cooperative model is the relay channel, it is not well suited for cognitive setups, since in the traditional relay channels, the relays do not have their own messages to be transmitted. This contradicts the main motivation of cognitive radio, where the purpose is to create an opportunity for the secondary users to share the medium with primary users to send their own message. Instead, the multiple access channel with generalized feedback [11] is an excellent model for cognitive multiple access channels, as it captures the overheard information and allows simultaneous transmission of information by all parties in addition to cooperation. The best known achievable rate region for this model was obtained in [11] for two users, using block Markov superposition encoding and backwards decoding, a technique which was later applied to a fading Gaussian setup in [12]. The simplest cognitive cooperative multiple access channel setup involving only one primary user, one secondary user and a common receiver was recently considered in [13], where the encoding strategy of [12] for the MACGF was modified by disabling cooperation from the primary user, to suit the cognitive nature of the channel. Under the assumption that the primary user-secondary user link is always better than the primary user-receiver link, [13] characterized the two-user achievable rates and optimized them in terms of the powers. However, this assumption is quite restrictive for practical scenarios, as fading can cause rapid fluctuations in relative channel gains. Moreover, in order to model the channel in larger networks involving many users, the locations of the users should also be taken into account, as the interactions of the users heavily depend on their positions.

In this paper, we consider a cognitive cooperative multiple access channel with $K$ primary and $K$ secondary users. We generalize the two user cooperation model of [13], which is valid for only a particular channel condition, to hold for any channel condition. We extend the two user cooperative model in [13] to $2 K$ users, by allowing pairwise partnering among primary and secondary users. The transmissions from different pairs are orthogonalized using OFDMA. Using an information theoretical framework, we characterize the rates achievable 
by each primary-secondary user pair, for both overlay and underlay scenarios, as a function of the users' powers and positions. We then solve the jointly optimal power allocation and partner selection problem, with two objectives: sum-rate maximization and maximization of secondary users' sum-rate. The solution is obtained by formulating the primary-secondary user pairing problem as a maximum weighted matching problem on a bipartite graph, where the weights are given by the optimized pairwise sum or secondary user rates. The optimum partnering strategies, and the resulting rate regions are analyzed by simulations.

\section{System Model}

We consider a fading cognitive cooperative multiple access channel with $K$ primary and $K$ secondary users. The users are to be divided into $K$ disjoint pairs, each consisting of one primary and one secondary user. Each pair is then assigned one of $K$ orthogonal subchannels using OFDMA, and users in a given pair communicate with each other, as well as with the receiver, over their assigned subchannel. This creates $K$ cognitive cooperative multiple access channels [13] in parallel, having one primary and one secondary user each. The primary and secondary users are randomly placed on a disk of radius $d$. The locations of the users, and the statistics of the channel gains among all users and the receiver are assumed to be known at the receiver. The receiver uses this information, and the fact that users in each group will use channel adaptive power control, to determine the optimal cooperating pair assignment, which is then fixed throughout the transmission. We assume that the subchannel assignment is fixed at the beginning of transmissions, and is not optimized instantaneously. Once the cooperating pairs are determined, it is sufficient to assume that each cooperating pair of users have only their own channel state information (CSI) (inter-user and user-receiver). Likewise, the receiver only needs the instantaneous CSI of the cooperating pairs, the CSI among non-cooperating primary-secondary users is not needed.

We denote by $p_{i}$ the $i$ th primary user, and by $s_{j}$ the $j$ th secondary user, where $i, j \in\{1, \ldots, K\}$. A sample system with $K=5$ is shown in Figure 1, along with one possible pairing strategy. Once the pairing is fixed, the received signals $Y_{r_{i j}}$ at the receiver, and $Y_{s_{j}}$ at the the secondary user for each cooperating pair $\left\{p_{i}, s_{j}\right\}$ can be written respectively as

$$
\begin{aligned}
Y_{r_{i j}} & =\sqrt{q_{p_{i} r} d_{p_{i} r}^{-\beta}} X_{p_{i}}+\sqrt{q_{s_{j} r} d_{s_{j} r}^{-\beta}} X_{s_{j}}+N_{r}, \\
Y_{s_{j}} & =\sqrt{q_{p_{i} s_{j}} d_{p_{i} s_{j}}^{-\beta}} X_{p_{i}}+N_{s_{j}},
\end{aligned}
$$

In (1)-(2), $X_{p_{i}}$ and $X_{s_{j}}$ denote the codewords sent by the primary and secondary users; $q_{p_{i} r}, q_{s_{j} r}$ and $q_{p_{i} s_{j}}$ denote the $p_{i}$ to receiver, $s_{j}$ to receiver and $p_{i}$ to $s_{j}$ channel power gains due to frequency flat fading; $N_{r}$ and $N_{s_{j}}$ denote the zero mean additive white Gaussian noise components at the receiver and $s_{j}$ respectively. Without loss of generality, the noise variances are assumed to be 1, as otherwise they can be buried in the channel gain statistics. The variables $d_{p_{i} r}, d_{s_{j} r}$, and $d_{p_{i} s_{j}}$ denote the $p_{i}$ to receiver, $s_{j}$ to receiver, and $p_{i}$ to $s_{j}$ distances respectively, and $\beta$ denotes the path loss exponent. We define the equivalent channel gains, $h_{p_{i} r}=q_{p_{i} r} d_{p_{i} r}^{-\beta}, h_{s_{j} r}=q_{s_{j} r} d_{s_{j} r}^{-\beta}$

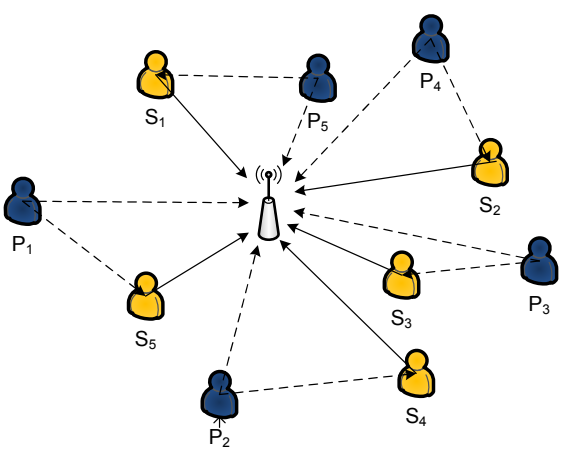

Fig. 1. Multi-user cooperative cognitive gaussian MAC.

and $h_{p_{i} s_{j}}=q_{p_{i} s_{j}} d_{p_{i} s_{j}}^{-\beta}$, hence absorbing the path loss in the fading statistics from now on.

Depending on whether or not the secondary users make use of their received signals $Y_{s_{j}},\{j=1, \ldots, K\}$, we have two possible operation modes: overlay (cooperative) or underlay. In the next section, we provide the encoding/decoding strategies for both modes and the resulting achievable rates, assuming the partnering is fixed. Later, we will formulate the optimal partnering problem.

\section{Cognitive Strategies and Achievable Rates}

\section{A. Overlay Mode}

This cooperation model is a generalization of the model introduced in [13], to $K$ parallel cognitive cooperative MACs, for arbitrary user locations and channel conditions. Let us assume that the partnering decisions are already made, and the pairs are fixed. The message generation then proceeds as in [13]: for each pair $\left\{p_{i}, s_{j}\right\}$, we divide the $p_{i}$ 's message into two submessages, i.e., $W_{p_{i}}=\left(W_{p_{i} r}, W_{p_{i} s_{j}}\right)$. The submessage $W_{p_{i} r}$ is the information sent directly to the receiver, and the submessage $W_{p_{i} s_{j}}$ is the part that can be decoded by both $s_{j}$ and the receiver. The message of $s_{j}, W_{s_{j}}$, is not partitioned. These messages are mapped to randomly generated codewords, whose entries are selected from unit Gaussian distributions, and are superposed after multiplication by channel adaptive powers, as follows [13]:

$$
\begin{aligned}
X_{p_{i}} & =\sqrt{P_{p_{i} r}(\mathbf{h})} X_{p_{i} r}\left(W_{p_{i} r}(b), W_{p_{i} s_{j}}(b-1)\right) \\
& +\sqrt{P_{p_{i} s_{j}}(\mathbf{h})} X_{p_{i} s_{j}}\left(W_{p_{i} s_{j}}(b), W_{p_{i} s_{j}}(b-1)\right) \\
& +\sqrt{P_{p_{i} c}(\mathbf{h})} C\left(W_{p_{i} s_{j}}(b-1)\right), \\
X_{s_{j}} & =\sqrt{P_{s_{j} r}(\mathbf{h})} X_{s_{j} r}\left(W_{s_{j}}(b), W_{p_{i} s_{j}}(b-1)\right) \\
& +\sqrt{P_{s_{j} c}(\mathbf{h})} C\left(W_{p_{i} s_{j}}(b-1)\right) .
\end{aligned}
$$

The tasks of component codewords $X_{s_{j} r}, X_{p_{i} r}, X_{p_{i} s}$ and $C$ follow directly from [12], [13]. The powers assigned to each codeword, which can be selected as functions of the available channel state information denoted by the channel state vector $\mathbf{h}=\left[h_{p_{i} r}, h_{p_{i} s_{j}}, h_{s_{j} r}\right]$, should satisfy the average power constraints,

$$
\begin{aligned}
P_{p_{i}}(\mathbf{h}) & =P_{p_{i} r}(\mathbf{h})+P_{p_{i} s_{j}}(\mathbf{h})+P_{p_{i} c}(\mathbf{h}) \\
P_{s_{j}}(\mathbf{h}) & =P_{s_{j} r}(\mathbf{h})+P_{s_{j} c}(\mathbf{h}) \\
E\left[P_{n}(\mathbf{h})\right] & \leq \bar{P}_{n} \text { where } n \in\left\{p_{i}, s_{j}\right\} .
\end{aligned}
$$


The main difference of the overlay encoding model in this paper from that of [13] is the way different channel states are handled. In [13], it was assumed that the channel states always satisfy $h_{p_{i} s_{j}}>h_{p_{i} r}$, and based on the properties of the optimal allocation for a two user cooperative MAC [14], $P_{p_{i} r}(\mathbf{h})$ was always set to zero. While this simplifies the achievable rate region considerably, and makes it concave in powers, it is not a valid assumption in a practical fading system with many users at random locations. Since each primary user can potentially be paired with each secondary user, for some user locations and/or fading states, the effective direct link gains are likely to be better than the inter-user link gains, after taking the distance dependent path loss into account. That is, one must partition the channel state space into two, i.e., $\phi_{1} \triangleq\left\{\mathbf{h}: h_{p_{i} s_{j}} \geq h_{p_{i} r}\right\}$, and $\phi_{2} \triangleq\left\{\mathbf{h}: h_{p_{i} s_{j}}<h_{p_{i} r}\right\}$. Then, following the findings in [14], we need

$$
\begin{array}{ll}
P_{p_{i} r}(\mathbf{h})=0, & \text { if } \mathbf{h} \in \phi_{1} \\
P_{p_{i} s_{j}}(\mathbf{h})=0, & \text { if } \mathbf{h} \in \phi_{2}
\end{array}
$$

As a result, denoting the rates of $p_{i}$ and $s_{j}$ by $R_{p_{i}}$ and $R_{s_{j}}$ respectively, assuming the receiver uses backwards decoding, and evaluating the rate constraints for each sub-message after conditioning on $\phi_{1}$ and $\phi_{2}$, the following rate region can be shown to be achievable,

$$
\begin{aligned}
& R_{p_{i}} \leq E\left\{\log \left(1+h_{p_{i} r} P_{p_{i} r}(\mathbf{h})\right) \mid \phi_{2}\right\} \operatorname{Pr}\left[\phi_{2}\right] \\
&+E\left\{\log \left(1+h_{p_{i} s_{j}} P_{p_{i} s_{j}}(\mathbf{h})\right) \mid \phi_{1}\right\} \operatorname{Pr}\left[\phi_{1}\right] \\
& R_{s_{j}}<E\left\{\log \left[1+h_{s_{j} r} P_{s_{j} r}(\mathbf{h})\right]\right\} \\
& R_{p_{i}}+R_{s_{j}} \leq \min \{E\{\log (A)\} \\
& E\left\{\log \left[1+h_{p_{i} r} P_{p_{i} r}(\mathbf{h})+h_{s_{j} r} P_{s_{j} r}(\mathbf{h})\right] \mid \phi_{2}\right\} \operatorname{Pr}\left[\phi_{2}\right] \\
&+E\left\{\log \left[1+h_{p_{i} s_{j}} P_{p_{i} s_{j}}(\mathbf{h})\right] \mid \phi_{1}\right\} \operatorname{Pr}\left[\phi_{1}\right] \\
&\left.+E\left\{\log \left[1+h_{s_{j} r} P_{s_{j} r}(\mathbf{h})\right] \mid \phi_{1}\right\} \operatorname{Pr}\left[\phi_{1}\right]\right\}
\end{aligned}
$$

where $A=1+h_{p_{i} r} P_{p_{i}}(\mathbf{h})+h_{s_{j} r} P_{s_{j}}(\mathbf{h})+$ $2 \sqrt{h_{p_{i} r} h_{s_{j} r} P_{p_{i} c} P_{s_{j} c}}$. Note that, an additional minimum rate requirement should be imposed on the primary user rate, to guarantee that it achieves at least its single user capacity with optimal power allocation [16], i.e.,

$$
R_{p_{i}} \geq E\left\{\log \left[1+P_{p_{i}}^{\star}(\mathbf{h}) h_{p_{i} r}\right]\right\} \triangleq B^{*}
$$

where $P_{p_{i}}^{\star}(\mathbf{h})$ is the optimal power level for single user transmission, obtained by waterfilling, satisfying the power constraint $E\left[P_{p_{i}}^{\star}(\mathbf{h})\right]=\bar{P}_{p_{i}}$; and $B^{*}$ is the resulting single user capacity of the primary user, without cooperation.

\section{B. Underlay Mode}

In the underlay mode, once the primary-secondary user pairs are fixed, secondary user $s_{j}$ operates at the corner of the two user MAC capacity region, which corresponds to the secondary user being decoded first by treating the $p_{i}$ signal as noise, while $p_{i}$ transmits at its single user optimum rate. Due to the orthogonality among each of the assigned distinct pairs, the achievable rates of each primary-secondary user pair, say $\left\{p_{i}, s_{j}\right\}$, is given by

$$
\begin{aligned}
& R_{p_{i}}=E\left[\log \left(1+h_{p_{i} r} P_{p_{i}}^{*}(\mathbf{h})\right)\right] \\
& R_{s_{j}}=E\left[\log \left(1+\frac{h_{s_{j} r} P_{s_{j} r}(\mathbf{h})}{1+h_{p_{i} r} P_{p_{i}}^{*}(\mathbf{h})}\right)\right]
\end{aligned}
$$

where $P_{p_{i}}^{*}(\mathbf{h})$ is the optimal single user power control, as before. In this mode, the minimum rate constraint for $p_{i}$ is automatically satisfied.

In the next section, we solve the jointly optimal power allocation and partner selection problem for both overlay, and underlay modes. For the overlay mode, we consider two separate objectives: sum rate maximization, which creates an extra incentive for the primary users to allow cooperation, and total secondary user rate maximization, which aims to assign as much rate for the cognitive users as possible, while still providing a maximum single-user rate guarantee for each primary user. For the underlay mode, the default objective is total secondary user rate maximization, as the primary user rates are fixed. Despite the lack of cooperation in the underlay mode, the partner selection is still important, as it determines the level of interference seen at the receiver for each pair.

\section{Jointly Optimum PARTNERING AND Power ALLOCATION}

So far, the rate regions achievable by pairs of primary and secondary users were characterized functions of the powers and channel states (and hence the locations), for a fixed pairing. However, the rates achievable by each pair, and hence, the total rate or the sum of secondary users' rates depend on which pairing strategy is used in the overall system. It is easy to check that there are a total of $K$ ! pairing strategies to choose from, and the selection of the optimal strategy depends on the rates achievable by each pair, which makes the power allocation and partnering problems coupled. Let the set of all $K$ ! pairing strategies be denoted by $\Gamma$, and let each feasible pairing strategy be denoted by $\Gamma_{l}$, where $l \in\{1, \ldots, K !\}$ The jointly optimal partnering and power allocation problem can be posed as,

$$
\begin{aligned}
\max _{\substack{\Gamma_{l} \in \Gamma \\
\mathbf{P}(\mathbf{h})}} & C_{\Gamma_{l}}\left(R_{p_{1}}, \ldots R_{p_{K}}, R_{s_{1}}, \ldots R_{s_{K}}\right) \\
\text { s.t. } & \left\{R_{p_{i}}, R_{s_{j}}\right\} \in R_{\text {feasible }}, \quad \forall(i, j) \in \Gamma_{l} \\
& E\left[P_{p_{i}}\right] \leq \bar{P}_{p_{i}}, E\left[P_{s_{j}}\right] \leq \bar{P}_{s_{j}}
\end{aligned}
$$

where, for sum rate maximization,

$$
C_{\Gamma_{l}}\left(R_{p_{1}}, \ldots R_{p_{K}}, R_{s_{1}}, \ldots R_{s_{K}}\right)=\sum_{(i, j) \in \Gamma_{l}} R_{p_{i}}+R_{s_{j}},
$$

and for secondary user total rate maximization,

$$
C_{\Gamma_{l}}\left(R_{p_{1}}, \ldots R_{p_{K}}, R_{s_{1}}, \ldots R_{s_{K}}\right)=\sum_{(i, j) \in \Gamma_{l}} R_{s_{j}} .
$$

The feasible set $R_{\text {feasible }}$ is defined as the rates satisfying constraints (10)-(13) for the overlay mode, and (14)-(15) for the underlay mode. $\mathbf{P}(\mathbf{h})$ is the vector of all power variables in the system. The key observation in solving (16) is that the joint optimization problem is separable into two stages: an inner 


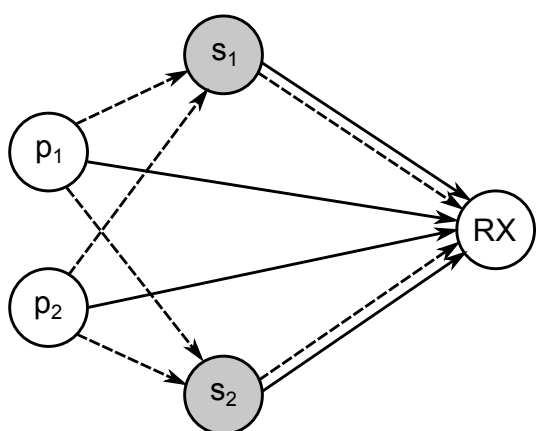

(a) A sample system with $K=2$ primary and secondary users.

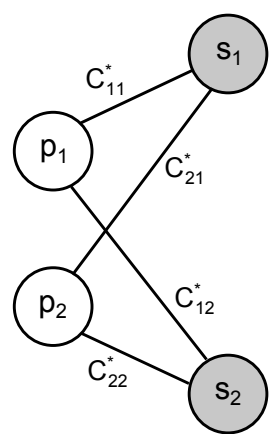

(b) Graph equivalent of the system in Fig. 2(a).
Fig. 2. Sample cognitive setup.

power optimization problem and an outer partnering problem, i.e.,

$$
\begin{array}{ll}
\max _{\Gamma_{l} \in \Gamma}, & \sum_{(i, j) \in \Gamma_{l}} \max _{\mathbf{P}_{i}(\mathbf{h}), \mathbf{P}_{j}(\mathbf{h})} C_{i, j}\left(R_{p_{i}}, R_{s_{j}}\right) \\
\text { s.t. } & \left\{R_{p_{i}}, R_{s_{j}}\right\} \in R_{\text {feasible }}, \quad \forall(i, j) \in \Gamma_{l} \\
& E\left[P_{p_{i}}\right] \leq \bar{P}_{p_{i}}, E\left[P_{s_{j}}\right] \leq \bar{P}_{s_{j}}
\end{array}
$$

where $C_{i, j}\left(R_{p_{i}}, R_{s_{j}}\right) \triangleq R_{p_{i}}+R_{s_{j}}$ for sum rate maximization, $C_{i, j}\left(R_{p_{i}}, R_{s_{j}}\right) \triangleq R_{s_{j}}$ for total secondary user rate maximization; and $\mathbf{P}_{i}(\mathbf{h}), \mathbf{P}_{j}(\mathbf{h})$ are the vectors of all power variables for $p_{i}$ and $s_{j}$ respectively. The separation in (19) is due mainly to the orthogonality of the user pairs, and the independence of each power optimization problem for each pair, once the partnering is fixed. For a more complete proof of this separation property, we refer the reader to the solution of a non-cognitive version of this problem, see [18]. In the following subsections, we propose efficient methods to solve the outer and inner optimization problems in (19).

\section{A. Optimum Selection of Pairing $\Gamma_{l}$}

Let us assume that the inner maximization problem in (19) is solved for all pairs $p_{i}, s_{j}$. There are $K^{2}$ such pairs, hence we can compute $K^{2}$ power optimized pairwise objective functions, $C_{i, j}^{*}, \forall i \in\{1, \ldots, K\}, j \in\{1, \ldots, K\}$. Then, viewing these objective functions as weights on a bipartite graph formed by exhausting all possible combinations of primary and secondary users, the partner selection problem turns into the well known maximum weighted matching problem from graph theory, which can be solved optimally by Edmond's algorithm [17] in polynomial $\left(O\left(K^{3}\right)\right)$ time. In Figure 2, the equivalence of a system with $K=2$ primary and secondary users, to a bipartite graph is shown. As a generalization, the bipartite graph representation of a system with an arbitrary number of users is shown in Figure 3. Therefore, to find the optimal partnering strategy in polynomial time, it suffices to find the optimal power allocation policies which maximize a given $C_{i, j}$, for all $p_{i}$ and $s_{j}$, which is performed in the next section.

\section{B. Power Optimization to Find $C_{i, j}^{*}$}

1) Overlay Mode: By introducing a parameter $\alpha \in\{0,1\}$, both sum rate and total secondary user rate optimization problems can be stated, and solved simultaneously:

$$
\sum_{\{i, j\} \in \Gamma_{l}} \max _{\mathbf{P}_{i}(\mathbf{h}), \mathbf{P}_{j}(\mathbf{h})} \alpha R_{p_{i}}+R_{s_{j}}
$$

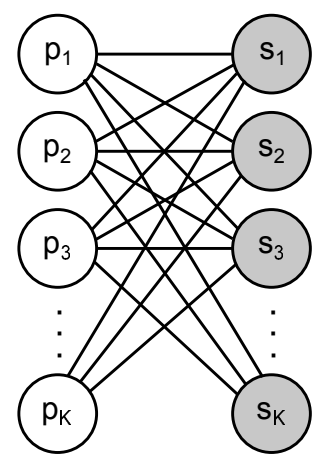

Fig. 3. Graph representation of any cooperative cognitive system.

$$
\begin{aligned}
\text { s.t. } R_{p_{i}} & \leq E\left\{\log \left(1+h_{p_{i} r} P_{p_{i} r}(\mathbf{h})\right) \mid \phi_{2}\right\} \operatorname{Pr}\left[\phi_{2}\right] \\
& +E\left\{\log \left(1+h_{p_{i} s_{j}} P_{p_{i} s_{j}}(\mathbf{h})\right) \mid \phi_{1}\right\} \operatorname{Pr}\left[\phi_{1}\right] \\
R_{s} & <E\left\{\log \left[1+h_{s_{j} r} P_{s_{j} r}(\mathbf{h})\right]\right\} \\
R_{p_{i}}+R_{s_{j}} \leq \min \{E\{\log (A)\} & E\left\{\log \left[1+h_{p_{i} r} P_{p_{i} r}(\mathbf{h})+h_{s_{j} r} P_{s_{j} r}(\mathbf{h})\right] \mid \phi_{2}\right\} \operatorname{Pr}\left[\phi_{2}\right] \\
& +E\left\{\log \left[1+h_{p_{i} s_{j}} P_{p_{i} s_{j}}(\mathbf{h})\right] \mid \phi_{1}\right\} \operatorname{Pr}\left[\phi_{1}\right] \\
& \left.+E\left\{\log \left[1+h_{s_{j} r} P_{s_{j} r}(\mathbf{h})\right] \mid \phi_{1}\right\} \operatorname{Pr}\left[\phi_{1}\right]\right\} \\
R_{p_{i}} & \geq B^{\star} \\
& E\left[P_{p_{i} r}(\mathbf{h})+P_{p_{i} s_{j}}(\mathbf{h})+P_{p_{c}}(\mathbf{h})\right] \leq \bar{P}_{p_{i}} \\
& E\left[P_{s_{j} r}(\mathbf{h})+P_{s c}(\mathbf{h})\right] \leq \bar{P}_{s_{j}} \\
& P_{p_{i} s_{j}}(\mathbf{h}), P_{p_{i} c}(\mathbf{h}), P_{s_{j} r}(\mathbf{h}), P_{s_{j} c}(\mathbf{h}) \geq 0
\end{aligned}
$$

Note that, by setting $\alpha=1$ in (20), we obtain the sum rate maximization for cognitive MAC, and by setting $\alpha=0$, we obtain the total secondary user rate maximization. While the rate constraints are significantly more involved compared to those in [13], due to the presence of two channel orderings, the solution proceeds similarly. First, by associating several Lagrange multipliers to the constraints in (21)-(27), we write the Lagrangian for our convex optimization problem:

$$
\begin{aligned}
\mathcal{L} & =\alpha R_{p_{i}}+R_{s_{j}} \\
& +\gamma_{1}\left\{E\left\{\log \left(1+h_{p_{i} r} P_{p_{i} r}(\mathbf{h})\right) \mid \phi_{2}\right\} \operatorname{Pr}\left[\phi_{2}\right]\right. \\
& \left.+E\left\{\log \left(1+h_{p_{i} s_{j}} P_{p_{i} s_{j}}(\mathbf{h})\right) \mid \phi_{1}\right\} \operatorname{Pr}\left[\phi_{1}\right]-R_{p_{i}}\right\} \\
& +\gamma_{2}\left\{E\left\{\log \left(1+h_{s_{j} r} P_{s_{j} r}(\mathbf{h})\right)\right\}-R_{s_{j}}\right\} \\
& +\gamma_{3}\left\{E\{\log (A)\}-R_{p_{i}}-R_{s_{j}}\right\} \\
& +\gamma_{4}\left\{E\left\{\log \left[1+h_{p_{i} r} P_{p_{i} r}(\mathbf{h})+h_{s_{j} r} P_{s_{j} r}(\mathbf{h})\right] \mid \phi_{2}\right\} \operatorname{Pr}\left[\phi_{2}\right]\right. \\
& +E\left\{\log \left[1+h_{p_{i} s_{j}} P_{p_{i} s_{j}}(\mathbf{h})\right] \mid \phi_{1}\right\} \operatorname{Pr}\left[\phi_{1}\right]
\end{aligned}
$$




$$
\begin{aligned}
& \left.+E\left\{\log \left[1+h_{s_{j} r} P_{s_{j} r}(\mathbf{h})\right] \mid \phi_{1}\right\} \operatorname{Pr}\left[\phi_{1}\right]-R_{p_{i}}-R_{s_{j}}\right\} \\
& +\gamma_{5}\left\{R_{p_{i}}-B^{\star}\right\} \\
& +\lambda_{1}\left\{P_{p_{i}}-E\left[P_{p_{i} r}(\mathbf{h})+P_{p_{i} s_{j}}(\mathbf{h})+P_{p_{i} c}(\mathbf{h})\right]\right\} \\
& +\lambda_{2}\left\{P_{s_{j}}-E\left[P_{s_{j} r}(\mathbf{h})+P_{s_{j} c}(\mathbf{h})\right]\right\} \\
& +\mu_{1} P_{p_{i} r}(\mathbf{h})+\mu_{2} P_{p_{i} s_{j}}(\mathbf{h})+\mu_{3} P_{p_{i} c}(\mathbf{h}) \\
& +\mu_{4} P_{s_{j} r}(\mathbf{h})+\mu_{5} P_{s_{j} c}(\mathbf{h}) .
\end{aligned}
$$

Taking the partial derivatives with respect to the power components of primary and secondary users, as well as the rate variables, and employing complementary slackness constraints in both cases, it is easy to show that the following KKT conditions are necessary and sufficient for optimality. For $\phi_{1}$,

$$
\begin{aligned}
& \lambda_{1} \geq\left(\gamma_{1}+\gamma_{4}\right) \frac{h_{p_{i} s_{j}}}{1+h_{p_{i} s_{j}} P_{p_{i} s_{j}}(\mathbf{h})}+\gamma_{3} \frac{h_{p_{i} r}}{A} \\
& \lambda_{2} \geq\left(\gamma_{2}+\gamma_{4}\right) \frac{h_{s_{j} r}}{1+h_{s_{j} r} P_{s_{j} r}(\mathbf{h})}+\gamma_{3} \frac{h_{s_{j} r}}{A}
\end{aligned}
$$

for $\phi_{2}$,

$$
\begin{aligned}
\lambda_{1} & \geq\left(\gamma_{1}+\gamma_{4}\right) \frac{h_{p_{i} r}}{1+h_{p_{i} r} P_{p_{i} r}(\mathbf{h})} \\
& +\gamma_{3} \frac{h_{p_{i} r}}{1+h_{p_{i} r} P_{p_{i} r}+h_{s_{j} r} P_{s_{j} r}} \\
\lambda_{2} & \geq\left(\gamma_{2}+\gamma_{4}\right) \frac{h_{s_{j} r}}{1+h_{s_{j} r} P_{s_{j} r}(\mathbf{h})} \\
& +\gamma_{3} \frac{h_{s_{j} r}}{1+h_{p_{i} r} P_{p_{i} r}+h_{s_{j} r} P_{s_{j} r}}
\end{aligned}
$$

and for $\phi_{1} \cup \phi_{2}$,

$$
\begin{aligned}
\lambda_{1} & \geq \gamma_{3} \frac{h_{p_{i} r} \sqrt{P_{p_{i} c}(\mathbf{h})}+\sqrt{h_{p_{i} r} h_{s_{j} r} P_{s_{j} c}(\mathbf{h})}}{A \sqrt{P_{p_{i} c}(\mathbf{h})}} \\
\lambda_{2} & \geq \gamma_{3} \frac{h_{s_{j} r} \sqrt{P_{s_{j} c}(\mathbf{h})}+\sqrt{h_{p_{i} r} h_{s_{j} r} P_{p_{i} c}(\mathbf{h})}}{A \sqrt{P_{s_{j} c}(\mathbf{h})}} \\
1 & =\gamma_{2}+\gamma_{3}+\gamma_{4} \\
\alpha+\gamma_{5} & =\gamma_{1}+\gamma_{3}+\gamma_{4}
\end{aligned}
$$

The constraints (29) - (34) are satisfied with equality, if the respective powers $P_{p_{i} r}(\mathbf{h}), P_{p_{i} s_{j}}(\mathbf{h}), P_{s_{j} r}(\mathbf{h}), P_{p_{i} c}(\mathbf{h}), P_{s_{j} c}(\mathbf{h})$ are positive. The solution of the optimal powers from these constraints requires rather lengthy derivations, and is omitted due to space limitations. It turns out that it is sufficient to search for at most two of the $\gamma_{i}$ 's which can be done iteratively. The details, and a closed form solution for optimal powers, are available in [19].

2) Underlay Mode: In underlay mode, the primary user employs single user waterfilling power control scheme. The secondary user adapts its own power according to primary users' transmit power. Therefore, only one Lagrange multiplier, say $\lambda_{s_{j}}$ is sufficient to compute each secondary user's power.
Solving (19) for (14)-(15),

$$
P_{s_{j}}(\mathbf{h})=\left(\frac{1}{\lambda_{s_{j}}}-\frac{1+h_{p_{i} r} P_{p_{i}}^{*}(\mathbf{h})}{h_{s_{j} r}}\right)^{+}
$$

Finally, for both overlay and underlay setups, the optimal power values can be used to compute the power optimized objective function, $C_{i, j}^{*}$.

\section{Simulation Results}

We consider a simulation environment with $K=20$ primary and secondary users randomly located according to a uniform distribution in a disk of radius $d=100 \mathrm{~m}$, where the receiver is located at the center. The path loss exponent and noise variances were selected as $\beta=2$ and $\sigma^{2}=1$, without loss of generality. Both sum rate maximizing and secondary user rate maximizing policies are obtained by using the developed optimal power control and partner selection algorithms. For benchmarking purposes, an underlay cognitive setup is also considered where the primary users allocate their powers according to single user water-filling, and the secondary users transmit at powers (37). The partners in the underlay mode are again selected by MWM.

In Fig. 4, matchings for a sample user distribution are presented, for $K=20$ pairs. Since the partner selection scales polynomially, it is practically possible to extend similar analysis to arbitrary $K$, and the choice here is only intended for a clearer visual demonstration of the matchings. From system throughput maximization point of view, sum rate maximizing power control policy with MWM achieves the highest throughput as expected. For secondary user rate maximization with MWM, the secondary users achieve a higher sum rate, in expense of decreased overall system sum rate. The underlay setup achieves a lower sum-rate, but is still acceptable for secondary user rate maximization, if one employs MWM to control the interference. It is instructive to observe that the primary and secondary users which are closest to each other and are at a moderately larger distance from the receiver, tend to benefit from cooperation, whereas if either the primary or secondary user is too close to the receiver, partnering and cooperation is not beneficial, and can be done randomly. This is observed by the optimum pairing structures in Figs. 4(a) and 4(b), and is further validated by plotting the achievable rate regions, corresponding to the partnering of Fig. 4(a), in Fig. 5. The rate regions closer to the axes in Fig. 5 correspond to either the primary, or the secondary user being too strong, and the rate regions in the center, which are zoomed in, correspond to the scenario when the secondary users can in fact surpass their single user capacities with power control, by cooperating with the primary users, while keeping the primary users at their single user optimum rates.

\section{CONCLUSION}

In this paper, we solved the joint power control and partnering problem for cognitive cooperative multiple access channels. We obtained sum-rate and sum-of-secondary-user-rates optimal policies, by use of convex optimization techniques in conjunction with techniques from graph theory, i.e., MWM. Simulation 
results showed that cooperation is especially beneficial for midrange primary-secondary user pairs, and the overlay setup with cooperation promises higher rates than the underlay setup.

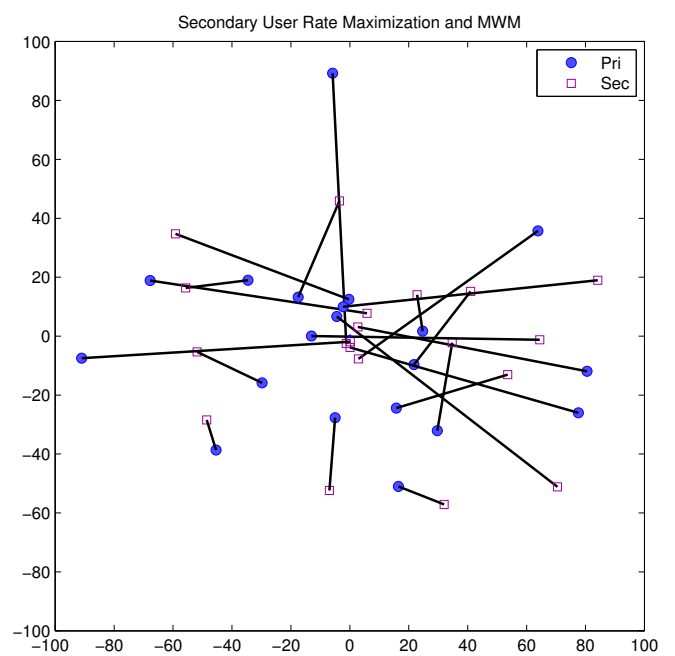

(a) Secondary user rate maximization, overlay with MWM: $\sum R_{s}=25.92, \quad \sum R_{p}+R_{s}=42.82$.

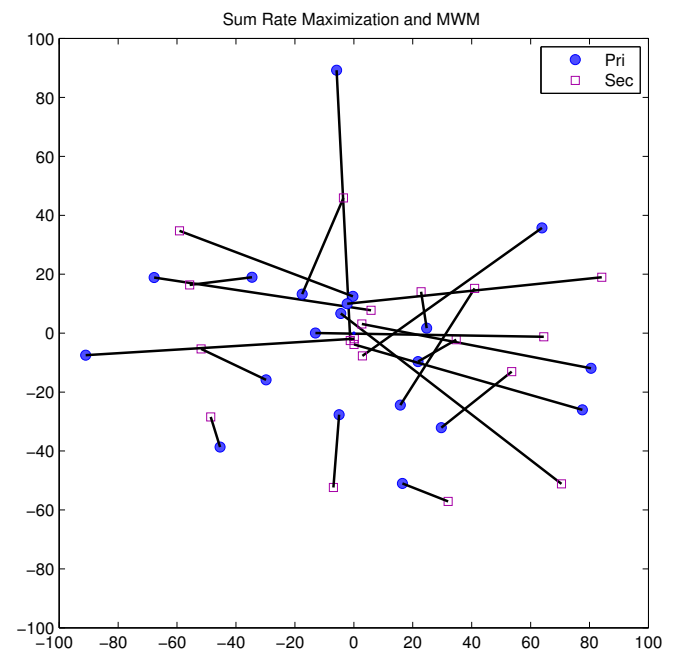

(b) Sum rate maximization, overlay with MWM: $\sum R_{s}=24.66, \quad \sum R_{p}+R_{s}=43.52$.

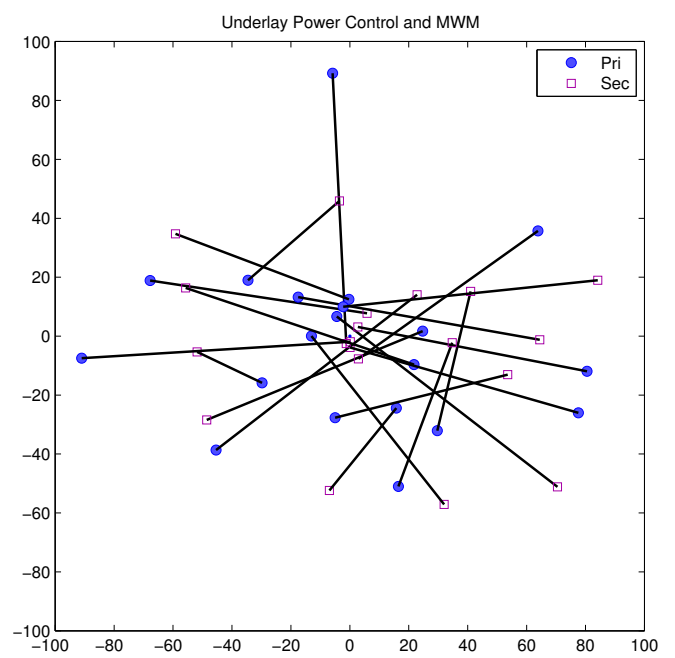

(c) Secondary user rate maximization, underlay with MWM: $\sum R_{s}=25.55, \quad \sum R_{p}+R_{s}=42.46$.

Fig. 4. Optimum partnerings obtained as a result of a sample simulation.

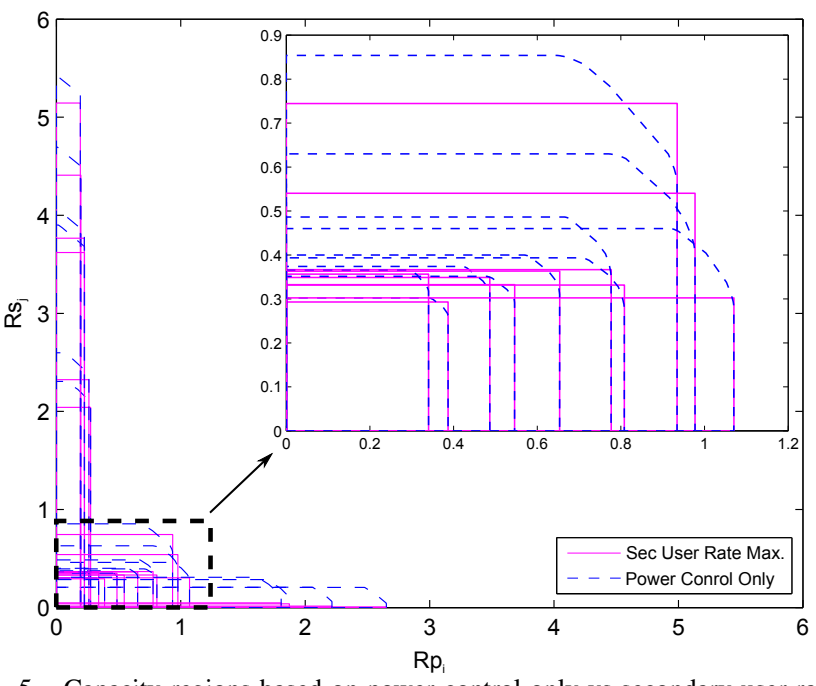

Fig. 5. Capacity regions based on power control only vs secondary user rates achievable by cooperation and power control.

\section{REFERENCES}

[1] E. C. Van der Meulen. "Three-Terminal Communication Channels." Adv. Appl. Prob., 3(1): 120-154, 1971.

[2] J. Mitola. "Cognitive radio: An integrated agent architecture for software defined radio." Ph.D. dissertation, KTH, Stockholm, Sweden, Dec. 2000.

[3] A. Goldsmith, S. A. Jafar, I. Maric and S. Srinivasa. "Breaking Spectrum Gridlock with Cognitive Radios: An Information Theoretic Perspective." Proceedings of the IEEE, 97(5): 894-914, May 2009.

[4] A. Jovicic and P. Viswanath. "Cognitive Radio: An InformationTheoretic Perspective." IEEE Trans. Inf. Theory, 55(9): 3945-3958, Sept. 2009.

[5] W. Wei, S. Vishwanath and A. Arapostathis. "Capacity of a Class of Cognitive Radio Channels." IEEE Trans. Inf. Theory, 53(11): 43914399, Nov. 2007.

[6] I. Maric, A. Goldsmith, G. Kramer and S. Shamai (Shitz). "On the capacity of interference channels with one cooperating transmitter." Eur. Trans. Telecommun., vol. 19, pp. 405-420, Apr. 2008.

[7] N. Devroye, P. Mitran V. Tarokh. "Achievable rates in cognitive radio channels." IEEE Trans. Inf. Theory, 52(5): 1813-1827, May 2006.

[8] D. Tuninetti. "On Interference Channel with Generalized Feedback (IFC-GF).” In Proc. ISIT 2007, pp. 2861-2865, Jun. 2007.

[9] S.H. Seyedmehdi, J. Jiang, Y. Xin and X. Wang. "An improved achievable rate region for causal cognitive radio." In Proc, ISIT 2009, pp. 611-615, Jul. 2009.

[10] Y. Cao, B. Chen and J. Zhang. "A New Achievable Rate Region for Interference Channels with Common Information." In Proc. IEEE WCNC 2007, pp. 2069-2073, Mar. 2007.

[11] F. M. J. Willems, E. C. van der Meulen and J. P. M. Schalkwijk. "An Achievable Rate Region for the MAC with Generalized Feedback." In Proc. Allerton Conference, Monticello, IL, Oct. 1983.

[12] A. Sendonaris, E. Erkip and B. Aazhang. "User Cooperation Diversity - Part I: System Description.” IEEE Trans. Commun., 51(11): 19271938, Nov. 2003.

[13] O. Kaya and M. Isleyen. "Power control in the cognitive cooperative multiple access channel." In Proc. CISS 2012, Princeton, NJ, March 2012.

[14] O. Kaya and S. Ulukus. "Power Control for Fading Cooperative MACs." IEEE Trans. Wireless Commun., 6(8): 2915-2923, Aug. 2007.

[15] J. Jiang and Y. Xin. "On the Achievable Rate Regions for Interference Channels With Degraded Message Sets.” IEEE Trans. Inf. Theory, 54(10): 4707-4712, Oct. 2008.

[16] A.J. Goldsmith and P.P. Varaiya. "Capacity of Fading Channels with Channel Side Information.” IEEE Trans. Inf. Theory, 43(6): 1986-1992, May 2010.

[17] H. N. Gabow. "An Efficient Implementation of Edmonds Algorithm for Maximum Matching on Graphs." J. ACM., 23(2): 221-234, Apr. 1976.

[18] S. Baksi, O. Kaya and T. Biyikoglu. "Optimal and Near-Optimal Partner Selection Algorithms in Cooperative OFDMA" In Proc. IEEE WCNC 2012, Paris, France, April 2012.

[19] S. Baksi. "Partner Selection and Resource Allocation in Singlecell, Multi-cell and Cognitive Cooperative Multiple Access Channels." M.Sc. thesis, Isik University, Istanbul, Turkey, June 2012. Available: http://www2.isikun.edu.tr/personel/onurkaya/baksithesis.pdf. 